5 World Health Organization. Guidance for National Tuberculosis Programmes on the Management of Tuberculosis in Children. 2nd Edn. WHO/HTM/TB/2014.03. Geneva, World Health Organization, 2014. http://apps.who.int/ medicinedocs/documents/s21535en/s21535en.pdf

6 Bonnet $\mathrm{M}$, Bastard $\mathrm{M}$, du Cros $\mathrm{P}$, et al. Identification of patients who could benefit from bedaquiline or delamanid: a multisite MDR-TB cohort study. Int J Tuberc Lung Dis 2016; 20: 177-186.

7 Pontali E, Sotgiu G, D'Ambrosio L, et al. Bedaquiline and multidrug-resistant tuberculosis: a systematic and critical analysis of the evidence. Eur Respir J 2016; 47: 394-402.

8 Esposito S, D'Ambrosio L, Tadolini M, et al. ERS/WHO Tuberculosis Consilium assistance with extensively drug-resistant tuberculosis management in a child: case study of compassionate delamanid use. Eur Respir J 2014; 44: $811-815$.

9 Tiberi S, De Lorenzo S, Centis R, et al. Bedaquiline in MDR/XDR-TB cases: first experience on compassionate use. Eur Respir J 2014; 43: 289-292.

10 Guglielmetti L, Le Dû D, Jachym M, et al. Compassionate use of bedaquiline for the treatment of multidrug-resistant and extensively drug-resistant tuberculosis: interim analysis of a French cohort. Clin Infect Dis 2015; 60: $188-194$.

11 Lessem E, Cox H, Daniels C, et al. Access to new medications for the treatment of drug-resistant tuberculosis: patient, provider and community perspectives. Int J Infect Dis 2015; 32: 56-60.

12 World Health Organization. Companion Handbook to the WHO Guidelines for the Programmatic Management of Drug-resistant Tuberculosis. WHO/HTM/TB/2014.11. Geneva, World Health Organization, 2014. http://apps. who.int/iris/bitstream/10665/130918/1/9789241548809_eng.pdf

13 Lachâtre M, Rioux C, Dû DL, et al. Bedaquiline plus delamanid for XDR tuberculosis. Lancet Infect Dis 2016; 16: 294.

14 Tadolini M, Lingtsang RD, Tiberi S, et al. First case of extensively drug-resistant tuberculosis treated with both delamanid and bedaquiline. Eur Respir J 2016; in press [DOI: 10.1183/13993003.00637-2016].

15 Matteelli A, D'Ambrosio L, Centis R, et al. Compassionate and optimum use of new tuberculosis drugs. Lancet Infect Dis 2015; 15: 1131-1132.

16 Nachman S, Ahmed A, Amanullah F, et al. Towards early inclusion of children in tuberculosis drugs trials: a consensus statement. Lancet Infect Dis 2015; 15: 711-720.

\title{
Variation in policy and practice of adolescent tuberculosis management in the WHO European Region
}

\author{
To the Editor:
}

Tuberculosis (TB) is a major public health concern worldwide. The World Health Organization (WHO) estimates that 9.6 million people fell ill with $\mathrm{TB}$ and 1.5 million people died of $\mathrm{TB}$ in 2014 . Of an estimated total of 1 million children with TB, 140000 children died [1]. The risk of progression to TB is highest for children $<5$ years of age. There is a comparatively low risk for those 5-10 years of age, after which the risk of progression to TB increases at the onset of puberty to an adult level of 5-10\% [2] as well as the chance of developing an adult-type TB accompanied by increased infectiousness [3]. However, adolescents form a particularly vulnerable group with psychosocial challenges distinct from adults and the usual challenges of transition from paediatric to adult health service provision. Puberty may affect health behaviour and have a significant impact on therapy compliance [4], requiring specific approaches usually not provided by paediatric or adult health services [5].

The current guidelines for childhood TB cover children from 0 to 14 years of age but do not specifically deal with adolescents aged 10-18 years $[5,6]$. WHO surveillance guidelines recommend to record and report two age bands (0-4 years and 5-14 years) [5], and thus specific epidemiological information on adolescents is lacking. To gain more insight in TB management among adolescents, the WHO European Regional Task Force on childhood TB conducted a survey among the 53 member states of the WHO European Region [7]. In January 2015, all national TB programme managers and designated childhood TB focal points were asked to provide information on the existence and nature of policies and practical management of adolescents with TB in their country. Responses received by April 30, 2015 were analysed using Excel 2010 (Microsoft, Redmond, WA, USA). 28 (53\%) countries responded to the first part of the questionnaire on policy and 20 countries also completed the questions on management of adolescents with TB. The relevant results are summarised in table 1. 
TABLE 1 Results from the questionnaire on the sections policy, treatment and prevention

High-burden countries Low-burden countries

Total countries

\begin{tabular}{|c|c|c|c|}
\hline \multicolumn{4}{|l|}{ Country income group } \\
\hline Middle income & $7 / 9(78 \%)$ & $5 / 19(26 \%)$ & $12 / 28(43 \%)$ \\
\hline \multicolumn{4}{|l|}{ Policy } \\
\hline Countries that expect adolescents to have increased risk of getting TB & 4/9 (44\%) & $3 / 19(16 \%)$ & $7 / 28(25 \%)$ \\
\hline Countries that feel the need for specific guidelines for adolescents & $3 / 9(33 \%)$ & $5 / 19(26 \%)$ & $8 / 28(29 \%)$ \\
\hline $\begin{array}{l}\text { Adolescents with drug-susceptible TB that are allowed to attend school } \\
\text { when smear and culture negative }\end{array}$ & $2 / 9(22 \%)$ & $15 / 19(79 \%)$ & $17 / 28(61 \%)$ \\
\hline $\begin{array}{l}\text { Countries that initiate ambulatory treatment for adolescents as soon } \\
\text { as possible }\end{array}$ & $2 / 8(25 \%)$ & $5 / 12(42 \%)$ & $7 / 20(35 \%)$ \\
\hline Countries that hospitalise adolescents during entire treatment period & $2 / 8(25 \%)$ & $0 / 12(0 \%)$ & $2 / 20(10 \%)$ \\
\hline Preventive therapy for exposed adolescents & $7 / 8(88 \%)$ & $10 / 12(83 \%)$ & $17 / 20(85 \%)$ \\
\hline Preventive therapy for adolescents in contact with a DR index case & $1 / 8(13 \%)$ & $1 / 12(8 \%)$ & $2 / 20(10 \%)$ \\
\hline Routine screening of TB disease in asymptomatic adolescents & $3 / 8(38 \%)$ & $3 / 12(25 \%)$ & $6 / 20(30 \%)$ \\
\hline
\end{tabular}

Six $(21 \%)$ countries reported a designated policy on adolescent TB. The age bands defining adolescents in these countries ranged between 14 and 19 years. In nine (32\%) countries, adolescents are managed as children and in 13 (46\%) countries as adults from the average age of 15 years (range 14-16 years). Only $25 \%$ of the countries perceive adolescents as a specific group with a high risk of TB and only $29 \%$ express a need for specific guidance.

All countries treat adolescents with the standard regimen ( 2 months of isoniazid, rifampicin, pyrazinamide and ethambutol, followed by 4 months of isoniazid and rifampicin) [5] for drug-susceptible TB and second-line drugs for confirmed drug-resistant (DR)-TB. Eight (42\%) countries also treat adolescents with clinical TB and recent contact with a DR-TB index case with second-line drugs.

Seven $(35 \%)$ countries provide early ambulatory care. Six (30\%) countries hospitalise adolescents for an average period of 45 days (118 days for DR-TB), five (25\%) while the adolescent is infectious and two (10\%) countries during the entire treatment period. When ambulatory care is provided, treatment supervision during the intensive phase is often the combined responsibility of caretakers and nurses who provide support through home or school visits. The responsibility is often shifted to the caretaker during the continuation phase. In 17 (61\%) countries, adolescents with noninfectious TB can attend school while still on treatment, five countries provide education on hospital or sanatorium premises and in three countries, no arrangements are made to continue school education.

Most countries follow WHO recommendations for contact investigation [6, 8]. Adolescents in the household of an index case (100\%), close nonhousehold contacts (95\%) and casual contacts (80\%) were eligible for contact investigation in most countries. 17 (85\%) countries test adolescent TB contacts for latent tuberculosis infection (LTBI) and provide preventive therapy to adolescents with LTBI or exposed to TB.

Six countries conduct routine screening for TB disease in asymptomatic adolescents starting at the average age of 15 years (range 11-18 years) while WHO recommends routine screening only for high-risk groups and geographically defined subpopulations with extremely high levels of undetected TB (1\% prevalence or higher) $[5,9]$.

The specific challenges relating to the management of $\mathrm{TB}$ in adolescents mentioned by the respondents are: lack of awareness in healthcare workers of the particular needs of adolescents with TB resulting in poor treatment adherence; diagnosis and follow-up of TB in adolescent migrants; inappropriateness of paediatric or adult TB facilities for adolescents; and lack of knowledge of the safety and effectiveness of second- and third-line drugs in adolescents with DR-TB.

This is the first survey of the current policies and practices on TB management in adolescents in the WHO European Region. The overall response rate in this survey was 53\%. Response rates from 
high-burden countries and low-burden countries were $64 \%$ and $49 \%$, respectively. Response rates from high-income and middle-income countries were $47 \%$ and $63 \%$, respectively $[1,10]$. The results show that practices vary, few countries have specific policies and guidelines for the management of $\mathrm{TB}$ in adolescents, and those that have may not be adequate for addressing the particular challenges. Most countries did not express a need for policies and guidelines for adolescents, because of a perceived low incidence and because they apply similar main principles for case management in adults or children.

We conclude that access to diagnosis and treatment is available for all adolescents with TB in the countries responding to our survey but there is a lack of policies that adequately deal with the unique challenges related to adolescence such as age group-sensitive support. In some countries, adolescents are still kept away from their families and friends for prolonged periods, thereby inducing stigma and social isolation. Adolescents should be treated in an ambulatory fashion and be allowed to continue their education as soon as can be allowed in view of infection control, their clinical condition and the availability of treatment support, as recommended by current international guidelines $[5,11,12]$. Evidence is needed on conditional or compassionate use of new medicines (bedaquiline and/or delamanid) for children including adolescents with extremely severe clinical conditions and when to recourse to other second-line drugs $[13,14]$.

The challenges and needs of adolescents with TB are similar worldwide, and must be addressed more effectively by all stakeholders [15]. Countries should be encouraged to monitor and evaluate the TB situation and case management of adolescents as a specific group. Specific epidemiological information on the occurrence and management of TB in the age group of 10-18 years, on a global as well as a country level, would provide more insight in the particular challenges and can contribute to guidance for age group-sensitive approaches to adolescents with TB.

@ERSpublications

Guidelines specifically addressing adolescent tuberculosis are needed for uniform and evidencebased management http://ow.ly/BMm63015LcY

Nick Blok ${ }^{1}$, Martin van den Boom ${ }^{2}$, Connie Erkens ${ }^{1}$, Masoud Dara $\odot^{2}$ and Susan van den Hof ${ }^{1,3}$

${ }^{1}$ KNCV Tuberculosis Foundation, The Hague, The Netherlands. ${ }^{2}$ World Health Organization, WHO Regional Office for Europe, Copenhagen, Denmark. ${ }^{3}$ Dept of Global Health, Academic Medical Center, and Amsterdam Institute for Global Health and Development, Amsterdam, The Netherlands.

Correspondence: Nick Blok, KNCV Tuberculosis Foundation, Benoordenhoutseweg 46, The Hague, 2596 BC, The Netherlands. E-mail: nick.blok@kncvtbc.org

Received: Feb 122016 | Accepted after revision: May 242016 | First published online: July 282016

This is one of a selection of articles published as ERJ Open papers, as part of an initiative agreed between the European Respiratory Society and the World Health Organization.

Conflict of interest: None declared.

\section{References}

1 World Health Organization. Global Tuberculosis Report. www.who.int/tb/publications/global_report/en/ Date last accessed: December 21, 2015. Date last updated: 2015.

2 Marais BJ, Gie RP, Schaaf HS, et al. The clinical epidemiology of childhood pulmonary tuberculosis: a critical review of literature from the pre-chemotherapy era. Int J Tuberc Lung Dis 2004; 8: 278-285.

3 Weber HC, Beyers N, Gie RP, et al. The clinical and radiological features of tuberculosis in adolescents. Ann Trop Paediatr Int Child Health 2000; 20: 5-10.

4 Taddeo D, Egedy M, Frappier J-Y. Adherence to treatment in adolescents. Paediatr Child Health 2008; 13: 19-24.

5 World Health Organization. Guidance for National Tuberculosis Programmes on the Management of Tuberculosis in Children. www.who.int/tb/publications/childtb_guidelines/en/ Date last accessed: September 2, 2015. Date last updated: 2014.

6 World Health Organization. Guidelines on the Management of Latent Tuberculosis Infection. www.who.int/tb/ publications/ltbi_document_page/en/ Date last accessed: September 2, 2015. Date last updated: 2015.

7 World Health Organization. Member States of the WHO European Region. www.euro.who.int/en/countries Date last accessed: January 15, 2016. Date last updated: 2016.

8 van der Werf MJ, Langendam MW, Sandgren A, et al. Lack of evidence to support policy development for management of contacts of multidrug-resistant tuberculosis patients: two systematic reviews. Int J Tuberc Lung Dis 2012; 16: 288-296.

9 World Health Organization. Systematic Screening for Active Tuberculosis: Principles and Recommendations. www.who.int/tb/tbscreening/en/ Date last accessed: December 21, 2015. Date last updated: 2013.

10 World Bank. Country and Lending Group. http://data.worldbank.org/about/country-and-lending-groups\#Lower_ middle_income Date last updated: 2015.

11 World Health Organization. Companion Handbook to the 2011 WHO Guidelines for the Programmatic Management of Multidrug-resistant Tuberculosis. www.who.int/tb/publications/pmdt_companionhandbook/en/ Date last accessed: December 21, 2015. Date last updated: 2015. 
12 Fitzpatrick MC, Floyd K. A systematic review of the cost and cost effectiveness of treatment for multidrugresistant tuberculosis. PharmacoEconomics 2012; 30: 63-80.

13 Esposito S, D’Ambrosio L, Tadolini M, et al. ERS/WHO Tuberculosis Consilium assistance with extensively drug-resistant tuberculosis management in a child: case study of compassionate delamanid use. Eur Respir J 2014; 44: 811-815.

14 Tadolini M, Dolma Lingtsang R, Tiberi S, et al. First case of extensively drug-resistant tuberculosis treated with both delamanid and bedaquiline. Eur Respir J 2016 [in press DOI: 10.1183/13993003.00637-2016].

15 Sandgren A, Cuevas LE, Dara M, et al. Childhood tuberculosis: progress requires an advocacy strategy now. Eur Respir J 2012; 40: 294-297.

\section{Efficacy and tolerability of ethionamide versus prothionamide: a systematic review}

To the Editor:

To treat multidrug-resistant tuberculosis (MDR-TB), the World Health Organization recommends to include, during the intensive phase of treatment, at least a parenteral agent, a later-generation fluoroquinolone, ethionamide (Eth) (or prothionamide (Pth)), cycloserine (Cs) or p-aminosalicylic acid (PAS) if Cs cannot be used, and pyrazinamide (Pzd) (which is not considered among the aforementioned four probably effective drugs) $[1,2]$. In particular, among the four drugs likely to be effective, at least two essential or "core" drugs (one with a good bactericidal and one with a good sterilising activity) and two other "companion" drugs should be administered [3,4].

In most of the countries where drug susceptibility testing cannot be performed to guide treatment regimen design, standardised second-line treatment regimens are prescribed, based on kanamycin $(\mathrm{Km})$, levofloxacin (Lfx), Eth, Cs and Pzd. Although the regimen is built following the international recommendations, the outcomes remain poor globally $[5,6]$. Less than $50-70 \%$ of the cases, in fact, achieve treatment success $[5,6]$, resulting in insufficient control of MDR-TB. It is widely recognised that one frequent cause of poor outcome is treatment default, which is mostly due to the low tolerability of the antituberculosis drugs employed [7]. One of the less tolerated antibiotics is Eth, because of the serious and frequent gastric adverse events [8 9] or of hypothyroidism, which is frequently subclinical.

Eth and Pth are thionamide drugs, characterised by a structure similar to isoniazid (Inh). They inhibit the mycobacterial synthesis of mycolic acid through a specific action against the inhA product enoyl-acyl carrier protein reductase; thus, they can be classified as bactericidal. However, their metabolic process is poorly known and, therefore, it is difficult to understand the pathogenesis behind the occurrence of adverse events following their administration.

If the reasons behind the choice of Km, Lfx, Cs and Pzd are in general easy to explain (although they do not necessary represent the best choice), less clear is the reason why Eth is used in the majority of the programmes instead of Pth. For this reason, we have carried out a systematic review on the existing evidence focused on efficacy and tolerability of Eth versus Pth.

The search was performed using the search engine PubMed, without any time restriction. Only articles written in English, French, Spanish and Italian were selected. The following keywords were used to retrieve the scientific references related to the research question: "ethionamide", "prothionamide", "efficacy", "safety" and "tolerability".

Reviews, case reports, case series and letters were excluded. References of the selected articles were analysed in order to identify significant manuscripts not found by the search engine.

We adopted a simplified five-point checklist adapted from that of the Scottish Intercollegiate Guidelines Network to grade the quality of the scientific evidence. A PRISMA (Preferred Reporting Items for Systematic Reviews and Meta-Analysis) flowchart was used to summarise the search and selection process (figure 1).

We found only six articles addressing this issue, all of them published before 1970. The core information derived from these articles is summarised in table 1. In the "double blind" trial by Chambatтe et al. [10], published in 1965 , Pth was compared to Eth $\left(1 \mathrm{~g} \cdot\right.$ day $\left.^{-1}\right)$. Tolerability was reported to be very good by $62 \%$ 